\title{
A new theoretical approach for cancer prevention: a novel strategy of using anti-epileptic medicines to prevent tumour growth
}

\begin{abstract}
This article is aimed at investigating the possibilities of preventing tumour growth associated with cancer by the use of Anti-Epileptic Drugs. The number of studies on the topic is still limited in number, but more studies associated with the use of Anti-Epileptic Medicine in cancer prevention can provide a much needed breakthrough in the prevention of cancer and tumour growth. Success in this field will not only provide a new means by which to minimize or prevent metastasis but will also help in saving cost and time by using a drug already in use for a different function. Recent studies have shown the possibility of some anti-epileptic drugs in inhibiting the growth of cancer cells, this though faces some challenges since there have also been links that suggest, some of thisanti epileptic drugs can be carcinogenic to humans. The analyses and information collection leading to the mentioned supposition has been done by assessing previous literature in the topic and further strengthen the case in a bid to raise attention of the scientific communities with the hope of further research on the area to potentially come up with a new treatment for cancer.
\end{abstract}

Volume 8 Issue 6 - 2017

\author{
Mustafa Pehlivan \\ Pharmacist and Pharmaceutical Health Scientist (BSc), University \\ of Hertfordshire, Cyprus International University, Cyprus \\ Correspondence: Mustafa Pehlivan, Pharmacist and \\ Pharmaceutical Health Scientist (BSc), University of \\ Hertfordshire, Cyprus International University, Bahadirsok. No \\ 10 k.kaymakli, Lefkosa, Nicosia-Cyprus, Tel +905338647864, \\ Email mustafa.pehlivan@yahoo.com.tr
}

Received: October 10, 2017 | Published: October 13, 2017

\section{Study overview}

Epilepsy is neurological disorders that are usually exhibited by seizures. These seizures can be very short lived and barely detectable or they can be characterized by vigorous shaking that can last for a long period. The immediate trigger for epileptic seizures cannot be pinpointed despite the fact that they have the tendency to recur. Most cases of epilepsy are caused by unknown causes. The most common causes include brain tumours, birth defects caused by different teratogens this happen in what is referred to as epileptogenesis, stroke or injury to the brain. Genetic mutations may play a very small role in the occurrence of epilepsy. The cause of epileptic seizures can be traced to abnormal and excessive activity of the nerve cells in the brain cortex.Epilepsy can be controlled using medication in most cases. In most western countries, a substantial portion of the population use anti epileptic drugs, this is because the disease is a very common neurological disorder that may be chronic in nature. Due to this long term use of these drugsthere have been claims that they may be responsible for the occurrence of some forms of cancer in human beings.

Cancer on the other hand is a disorder characterized by uncontrolled cell proliferation leading to the growth of abnormal cellswhich have the ability of invasion and spreading into other parts of the body. Cancer manifests itself in the formation of malignant neoplasms. Cancer is the second deadliest disease in the number of fatalities. A lot of discussions have been brought forward on the possible relationship between epilepsy and cancer. The discussions revolve on the question whether there is an increased chance of cancer in people with epilepsy, or whether the use of antiepileptic drugs can be associated with promotion or protection against cancer. There are carcinogenic risks that may be attributed to long period usage of antiepileptic drugs.

\section{Epilepsy and cancer links and common points in mechanisms}

A number of studies have studies have been carried out to try and investigate the links that may exist between epilepsy and the occurrence of cancer. One of the triggering factors for this investigation was the observation that there was an increased mortality rate in cancer among people who were institutionalized suffering from epilepsy. The use of phenytoin and Phenobarbital as anti-epileptic diseases has been suggested to be the cause behind cancer occurrence in epileptic patients. The study showed that there was a correlation where there was an increased diagnosis of cancer the same year epilepsy was diagnosed. However there is no clear clinical association between cancer and epilepsy from the study, and the increased risk for cancer in the same year that epilepsy was diagnosed was most likely brought about by the clinical investigations carried out after the onset of epileptic seizures.

Voltage Gated Sodium Channels (VGSCs) are the other possible links between epilepsy and cancer. Voltage Gated Sodium Channels are considered as the molecular characteristics exhibited by excitable cells. The activation of these channels is triggered by depolarization of membranes and this generates sodium currents that are responsible for activating and initiating action potentials in muscle cells and neurons. ${ }^{1}$ Abnormal activities of the Voltage Gated Sodium Channels are considered to be the central cause for epileptic seizures. Upon hyper excitability these VGSCs are used in cell signalling. If this signalling rate exceeds a certain limit, then neuronal disruptions occurs and this leads to seizures associated with epilepsy. Mutation of Voltage Gated Sodium Channels has also been attributed as a cause of epilepsy in humans. Humans with cases of inherited epilepsy were found to have a mutation of the NaV1.1 channels. ${ }^{2}$

Voltage Gated Sodium Channels have also been found to be instrumental in the spread of cancer. Although this channels are characteristic of excitable cells it has been shown that non excitable cells also have the ability to express Voltage Gated Sodium Channel. ${ }^{1}$ This is manifested either in physiological conditions or in pathological conditions. By being able to involve in biological roles other than generating action potentials, the Voltage Gated Sodium Channels exhibit abnormal expressions that are pathological in nature in the tissues. Epithelial cancer cellshave been shown to be caused by abnormal activities of the VGSCs. In cancer cells the VGSCs 
are responsible for the regulation of the migrative, proliferative and invasive properties of these cells. Voltage Gated Sodium Channels are also responsible for overactive signalling of the cells observed in breast cancer.

The other possible shared link between epilepsy and cancer is the concentration of glucose in the body. An excessive intake of glucose leads to an increase in energy production. This energy is used by neurons and an excess in this energy increases the speed of neuronal and cell signalling. When this signalling exceeds a certain limit seizures occur. Seizures are characteristic of epilepsy and therefore glucose plays some role in this disorder. Epilepsy caused by hyper polarization shares a link with cancer since both can be attributed to excessive glucose intake.

Normal healthy cells undergo apoptosis which is the programmed cell death. Cancer cells on the other hand have come up with ways of evading this natural mechanism. Being able to avoid apoptosis by cancer requires a high amount of glucose. Unlike normal cells that can use other alternatives of energy source such as fats, cancer cells only depend on glucose as their sole energy source. When they lack glucose, they have mechanisms to produce glucose that is enough to make them survive without necessarily growing. Therefore excessive intake of glucose by the body aides in the multiplication of cancer cells in the body. These are some of the shared mechanisms that cancer and epilepsy share.

\section{Anti-epileptic medicines and how they stop or slow down tumor growth?}

Anti-epileptic medicines can be grouped into three groups depending on their mechanism of action. These three groups includes drugs that carry out their action by blocking neuronal ion channels, the other group of drugs carry out their function by facilitating gamma-aminobutryic action. ${ }^{3}$ The last group carries out their function in a mode that has not been resolved yet. There have been constant discussions on whether anti-epileptic drugs act as carcinogens or whether they play a role in the reduction of tumour growth responsible for cancer. In order to come up with conclusions on this, experiments are carried out on animals. Phenobarbitals have been implicated as being responsible for growth of liver tumours in tests done on rats. Phenobarbitals have also been associated with lung cancer in humans and this is in early epidemiological studies. They have also been implicated in the occurrence of brain tumours. ${ }^{3}$ The other commonly used group of anti-epileptic drugs are the phenytoin. Phenytoins have been thought to contain some carcinogenic properties. Three human cancers have been linked to have an increased chance of occurrence due to the use of this group of anti-epileptics. These areneuroblastoma, lymphoma and myeloma. However it is important to note that no consistent data or results have been collected to accurately confirm that the two group of drugs are cancer causing. Phenobarbitals and phenytoins are only thought to be possible carcinogenic agents, necessitating for more medical research on the subject. ${ }^{3}$

Valproate is the other group of anti-epileptic drugs. The drugs seem to be the possible hope for treatment against cancer using anti epileptics drugs. The drug has been shown to have antiproliferative properties against some type of cancer cells. They show this property when they are administered both in vivo and in vitro. ${ }^{4}$ The drug is used in humans when dealing with solid and haematological tumours. Valproate is made from valproic acid. A lot of study has been done on the effects of valproic acid on the growth of cancer cells such as glioblastoma and ovarian cancer cells. The anti-cancer properties exhibited by valproic acid is linked to its ability to inhibit histone deacetylase and also to act independently of hormones and other mechanisms such as multidrug protein resistance. ${ }^{5}$

Valproic acid shows a lot of promise in being used as an anticancer agent probably in association with other existing drugs. Tests done on human glioma cells using valproic acid have shown that the drug aided in inhibiting the proliferation of this cells when treated using a chemotherapeutic agent such as temozolomide. The continued use of valproic acid has also been shown to increase the sensitivity of tumour cells to radiotherapy. Although the mechanisms by which the drug gets to do this is unknown, there is a full consensus in the scientific world that valproic acid exhibits an antiproliferative action on glioma cells. The drug has also been tested and shown to produce synergistic cytotoxicity when used together with cisplastin. This has been effective against all ovarian carcinoma cells and also the acid has been shown to be capable of resensitizing cancer cells that had already acquired resistance against cisplastin. ${ }^{6}$ Therefore there already exists evidence that valproic acid used in treating epilepsy seizures can be repurposed as a drug against cancer.

Other anti-epileptic drugs such as Oxcarbazepine have also been shown to have some anti-cancer properties. Although it is important to note that the concentration at which this drug exhibits these properties is way higher than the concentration it is at as an anti-epileptic drug. Oxcarbazepinehas been shown to inhibit the growth of cancer cells. It has also been shown to have the ability to induce apoptosis of cancer cells as well as G2M arrest in these cells. ${ }^{7}$

\section{Mechanism of action in anti-epilepticdrugs and how they prevent the risk of tumor development}

\section{A. Sodium channel blockers}

Anti-epileptic drugs that work by interacting with voltage-gated sodium channels are referred as sodium channel blockers. Tonic clonic seizures are mostly treated using sodium channel blockers. ${ }^{2}$ Examples of this kind of drugs include carbamazepine, phenytoin, oxcarbazepine, lacosamide, and lamotrigine. The blocking action employed by sodium channel blockers is use-dependent. The dominant type of action potentials exhibited by sodium channel blockers is trains of high frequency. Individual action potentials that fire at low frequencies are also exhibited but in a less potent manner. Seizures have action potentials that ride on adepolarized plateau potential. Sodium channel blockers are able to inhibit seizures strongly because they display "voltage dependence" during their blocking action. Sodium channel blockers preferred mode of action is inhibiting seizures discharges in relation to ongoing neural activity of a normal nature [8]. With the use of sodium channel blockers, action potentials are unable to invade nerve terminals. Therefore, the release of diverse neuro transmitters like glutamate is inhibited. ${ }^{2}$

Sodium channel blockers have a binding site that overlaps the binding site of local anesthetics on sodium channels. The S6 segments of domains create this site I, II, and IV within the pore of the channel. When the sodium channel is in the inactive state, the sodium channel blockers bind to this site with a higher affinity. The sodium channel becomes stabilized when the drug is bound in its inactive state. During seizures, depolarized neurons fire rapidly and the sodium channels spend a significant amount of time in an inactive state. The bound drug becomes accumulated and consequently, become trapped when the channels are inactivated. ${ }^{1}$

\section{B. GABA Inhibition}

Gamma Amino Butyric Acid is the neurotransmitter associated with local inhibitory interneuron's and performs its action through 
GABAA receptors and the GABAB receptors. GABAA receptors are the targets for the anti-epileptic drugs while as $\mathrm{GABAB}$ receptors only act as activators of potassium channels and inhibitors of calcium channels, and it is important to note that they are not a target for any anti-epileptic drugs (Stephanie Z, 2009). This mode of inhibition is important in regulating the excitory neurons as well as the firing rate by these neurons. They also help in synchronizing the activity of local neurons and in this way they prevent the generation or onset of abnormal epileptic behavior. GABA inhibition is one key method anti-epileptic action (Stephanie Z, 2009).

Although valproic acid is an important anti-epileptic drug with anti-cancer properties, it is not yet clearly understood how it acts in the prevention of seizures in patients suffering from epilepsy. It is assumed that it has a number of targets, and the overall action on these targets is responsible for its therapeutic abilities. It is one of the exceptions to anti-epileptic medicines and has some anti-cancer properties and the potential to inhibit tumour growth associated with cancer in humans. ${ }^{9}$ Interestingly the drug is excluded from the GABA inhibitors list since it mostly functions as a GABA activator rather than as an inhibitor. There is more need to study the mechanism employed by valproic acid since there are arguments in some quarters that activation or synthesis of GABA results in an increased risk for cancer. This is backed up by suggestions that GABA receptors are virtually involved in every stage and aspect present in cell development. This can be proved by the fact that certain inhibitors of GABAA receptors such as picrotoxin, are essential in inhibition of some cancer cells growth like is the case in prostate cancer cells (Stephanie Z, 2009). GABAA Receptors have also been shown to increase cell proliferation in some cancer line cells such as gastric cancer cells. ${ }^{10,11}$

\section{Conclusion and possibility of future studies}

From the above discussions it is clear that further studies are needed on the viability of anti-epilepticdrugs use in the treatment of cancer. Current studies show that valproic acid can be used to control glioma cells in association with other chemotherapeutic agents, further studies should be done on other anti-epileptic drugs to see if this would be possible. There is also need for further studies on the mechanisms employed by oxcarbazepine against cancer cells and see if this can be replicated in other anti-epileptic drugs. Further studies should be done on both valproic acid and oxcarbazepine to see if they can be used in local treatment of cancer. There is also a need to study on the benefits and risks that can be attributed to the use of Multidrug therapy in the treatment of different cancer lines. Multidrug therapy may be the answer to many medical problems by utilizing more than one class of drug in treating given medical problem.

Anti-epileptic medicines have a potential of revolutionizing cancer treatment since they have some properties that aide in the inhibition of tumor growth. All that is needed is further studies on the already existing leads and this would become possible in the near future. This is fueled by the fact that oxcarbazepine and valproicacid are effective in inhibiting cancer cell growth and also aide in apoptosis and G2M arrest. The fact that both drugs act as anti-cancer agents at concentrations that are higher than the ones they possess as antiepileptic drugs means that there is a need to come up with ways that the drugs can be used in local treatment of cancer. Possible ways in which this can be surpassed is by use of the drugs as implants. This could also revolutionize the use of multidrug treatment in combating cancer, and this would be important since we would utilize already existing drugs and there would be no need to undergo the tedious and costly process involved in coming up with new drugs. Furthermore to try and combat the link between epilepsy and cancer it is important for epilepsy patients to be treated with drugs that showed no enhancement of cancer cell growth. Therefore anti-epileptic drugs can help reduce the economic burden that comes with the discovery of new drugs for cancer treatment, and it would still revolutionize the treatment of cancer as we know it today.

\section{Acknowledgments}

None.

\section{Conflicts of interest}

The author declares that he has no conflict of interest for this paper.

\section{References}

1. Nelson M, Yang M, Dowle AA, et al. The sodium channel-blocking antiepileptic drug phenytoin inhibits breast tumour growth and metastasis. Mol cancer. 2015;14(1):13

2. Takada M, Fujimoto M, Motomura $\mathrm{H}$, et al. Inverse association between sodium channel-blocking antiepileptic drug use and cancer: data mining of spontaneous reporting and claims databases. Int J Med Sci. 2016;13(1):48-59.

3. Lee CY, Lai HY, Chiu A, et al. The effects of antiepileptic drugs on the growth of glioblastoma cell lines. J Neurooncol. 2016;127(3):445-453.

4. Terranova-Barberio M, Roca MS, Zotti AI, et al. Valproic acid potentiates the anticancer activity of capecitabine in vitro and in vivo in breast cancer models via induction of thymidine phosphorylase expression. Oncotarget. 2016;7(7):7715-7731.

5. Shirsath N, Rathos M, Chaudhari U, et al. Potentiation of anticancer effect of valproic acid, an antiepileptic agent with histone deacetylase inhibitory activity, by the cyclin-dependent kinase inhibitor P27600 in human non-small-cell lung cancer cell lines. Lung Cancer. 2013;82(2):214-221.

6. Kwiecińska P, Wróbel A, Taubøll E, et al. Valproic acid, but not levetiracetam, selectively decreases HDAC7 and HDAC2 expression in human ovarian cancer cells. Toxicol lett. 2014;224(2):225-232.

7. Rundle-Thiele D, Head R, Cosgrove L, et al. Repurposing some older drugs that cross the blood-brainier and have potential anticancer activity to provide new treatment options for glioblastoma. Br J Clin Pharmacol. 2016;81(2):199-209.

8. Macdonald RL. Inhibitory synaptic transmission. In: Engel J, Pedley TA, (Eds.), Epilepsy: A Comprehensive Textbook. Lippincott-Raven Publishers: Philadelphia, USA; 1997. 1383-1391 p.

9. Federica Iannelli, Rita Lombardi, Biagio Pucci, et al. Repurposing of valproic acid and simvastatin combination as anticancer agents in prostate cancer: synergistic interaction with docetaxel and suppression of docetaxel resistance. Experimental and Molecular Therapeutics. 2016;76(14):4745.

10. Nanako Shiraishiet. Effects of $\gamma$-Aminobutyric Acid (GABA) on Proliferation of the Gastric Cancer Cell Line, KATO III. Bulletin of the Osaka Medical College. 2007;5(1):33-43.

11. Engel J, Pedley TA. Introduction: what is epilepsy. Epilepsy: A comprehensive textbook; 1997. 1-10 p. 